\title{
PERFIL DE INTERNAÇÕES DE IDOSOS EM UMA CLÍNICA DE NEUROCIÊNCIAS DE UM HOSPITAL PÚBLICO
}

Daiane Borges Queiroz ${ }^{* *}$, Lorena Cajaiba de Oliveira***, Claudineia Matos de Araújo****, Luciana Araújo dos Reis*

\footnotetext{
Autora para correspondência: Luciana Araújo dos Reis - lucianauesb@yahoo.com.br

* Doutora em Ciências da Saúde/UFRN. Docente da Universidade Estadual do Sudoeste da Bahia e da Faculdade Independente do Nordeste

** Mestranda em Enfermagem e Saúde Universidade Estadual do Sudoeste da Bahia (UESB), docente do Curso de Fisioterapia da Universidade Estadual do Sudoeste da Bahia

*** Fisioterapeuta pela Universidade Estadual do Sudoeste da Bahia

**** Graduanda em Fisioterapia pela Universidade Estadual do Sudoeste da Bahia
}

\section{Resumo}

Esta pesquisa tem por objetivo descrever o perfil epidemiológico de idosos hospitalizados numa Clínica de Neurociências num hospital da rede pública no interior da Bahia. Estudo de caráter descritivo exploratório com delineamento transversal e abordagem retrospectiva de dezembro de 2011 a dezembro de 2012, com revisão do prontuário de 135 idosos atendidos numa Clínica de Neurociências num hospital público no interior da Bahia. Os resultados indicam que dos 135 prontuários avaliados a média de idade é igual a $77,23( \pm 10,28)$ anos, com $57,7 \%$ do sexo feminino e o problema de saúde mais identificado foi o Acidente Vascular Encefálico, com 84,4\% dos idosos acometidos. Em relação às patologias associadas verificou-se maior distribuição de Hipertensão Arterial Sistêmica $(31,9 \%)$, Diabetes Melitus associada à Hipertensão Arterial Sistêmica (13,3\%) e Hipertensão Arterial Sistêmica associada à Cardiopatia (4,4\%). Conclui-se que o perfil epidemiológico dos idosos avaliados é predominantemente do sexo feminino, sendo a patologia de maior frequência o Acidente Vascular Encefálico.

Palavras-chave: Idoso; Epidemiologia; Acidente Vascular Cerebral; Envelhecimento; Neurociências. 


\title{
ELDERLY ADMISSIONS PROFILE IN A PUBLIC HOSPITAL NEUROSCIENCE CLINIC
}

\begin{abstract}
This research aims to describe the epidemiological profile of hospitalized elderly in a neuroscience clinic from a public hospital in Bahia. It's an exploratory descriptive study with cross-sectional and retrospective approach ranging from December 2011 to December 2012, with review of medical records of 135 elderly treated in a neuroscience clinic at a public hospital in Bahia. The results indicate that among the 135 charts studied the average age is equal to $77.23( \pm 10.28)$ years, with $57.7 \%$ of females. The most frequent health problem identified was the stroke, with a prevalence of $84.4 \%$ among the elderly patients. Regarding comorbidities, there was greater distribution of hypertension (31.9\%), diabetes mellitus associated with hypertension (13.3\%) and arterial hypertension associated with systemic heart disease (4.4\%). We conclude that the epidemiological profile of the elderly evaluated is predominantly female, with the most frequent pathology being the stroke.
\end{abstract}

Keywords: Aged; Epidemiology; Stroke; Aging; Neurosciences.

\section{INTRODUÇÃO}

Um dos fenômenos sociais que acomete o Brasil e o mundo é o envelhecimento populacional, caracterizado por uma porção significativa da população que tem atingido e permanecido por mais tempo na faixa etária acima de 60 anos.(1) Esse processo de transição ocorreu de forma insidiosa e lenta, sendo possível graças às melhores condições sociais e de saneamento, além do uso de antibióticos e de vacinas. ${ }^{(2)}$

O processo de envelhecimento como modificações morfológicas, funcionais, bioquímicas e psicológicas que determinam a perda progressiva das capacidades de adaptação do indivíduo ao meio ambiente, tornando-o mais vulnerável e aumentando a incidência de processos patológicos. ${ }^{(1)}$ Os idosos são mais susceptíveis a patologias, pois possuem uma capacidade diminuída de responder ao estresse imposto pelo meio. Há, dessa forma, uma capacidade funcional reduzida e sobrecarga dos mecanismos de controle homeostáticos, servindo como substrato fi- siológico para influência da idade na apresentação da doença, da resposta ao tratamento proposto e das complicações seguintes. ${ }^{(3)}$

O cuidado ao idoso é, por definição, abrangente, envolvendo intervenções em diferentes níveis de atenção e espaços institucionais, incluindo unidades de internamento, hospital-dia e centros para cuidado prolongado. O novo perfil das doenças gera uma frequente necessidade de hospitalização, principalmente dado a cronicidade e complicações advindas das patologias que acometem esse grupo populacional, somado as consequências que a permanência imóvel por muito tempo em leitos hospitalares podem ocasionar, como a morte, por exemplo. ${ }^{(1,4,5)}$

$\mathrm{Na}$ velhice o risco de hospitalização em situações agudas e crônicas aumenta, pois os idosos tendem a apresentar multipatologias, constituindo, portanto, o grupo etário da população que mais utiliza os cuidados hospitalares. A internação 
hospitalar para os idosos apresenta diversos, e um deles consiste que o envelhecimento enfraquece mecanismos fisiológicos protetores. Os principais motivos de internação característicos desse grupo populacional são as doenças do aparelho circulatório, respiratório, digestivo, geniturinário, do olho e anexos, e neoplasias. ${ }^{(6)}$

A crescente necessidade de assistência e tratamento de uma população que envelhece, exige políticas adequadas, sendo que a falta dessas pode causar importante aumento dos custos. As políticas que propiciam a saúde durante toda a vida promoção da saúde e prevenção de doenças - a tecnologia de assistência, os cuidados para a reabilitação, os serviços de saúde mental, a promoção dos modos de vida saudáveis e ambientes propícios, podem reduzir os níveis de incapacidade associados à velhice e permitir obter economias orçamentárias. ${ }^{(5)}$

Reconhecendo as necessidades da população aqui em questão e sabendo-se da nova configuração social do cenário brasileiro, foram instituídas políticas que asseguram os direitos da pessoa idosa, como a Política Nacional do Idoso, que foi regulamentada pela Lei $n^{\circ} 8.842$, de 4 de janeiro de 1994 - Cap. IV, art. 10, dispondo sobre os cuidados de saúde direcionados a este grupo populacional; e o Estatuto do Idoso, lei $n^{\circ} 10.741$ de $1^{\circ}$ de outubro de 2003, que prescreve diretrizes para o cuidado, com objetivos de prevenção e manutenção da saúde deste grupo populacional.(7)

Frente ao novo perfil da população brasileira, com alterações no cenário epidemiológico e suas demandas especificas, fazem-se necessários mais estudos que conheçam e compreendam as particularidades da assistência ao indivíduo idoso e consequentemente, sejam criadas estratégias a fim de aprimorar os serviços de saúde destinados a esse público que configura o desenho nacional, assim, o presente estudo objetiva descrever o perfil epidemiológico de idosos hospitalizados numa Clínica de Neurociências num hospital da rede pública no interior da Bahia.

\section{MATERIAIS E MÉTODOS}

A pesquisa apresenta caráter descritivo exploratório com delineamento transversal e abordagem retrospectiva de dezembro de 2011 a dezembro de 2012 , e se caracteriza por observar, registrar e analisar o perfil epidemiológico dos idosos atendidos no Centro de Neurociências de um Hospital público no interior da Bahia, sem manipulá-los, realizando descrições precisas da situação e descobrindo as relações existentes entre os elementos componentes da pesquisa.

O local de estudo foi a Clínica de Neurociências de um hospital público no interior da Bahia, e a população alvo do estudo foi representada pelo prontuário de 135 idosos hospitalizados na Clínica de Neurociências do referido hospital.

A coleta dos dados foi obtida através do acesso às informações armazenadas nos prontuários dos pacientes. Foi utilizada uma ficha adaptada contendo as informações de interesse neste estudo e disponíveis nos prontuários dos idosos, constituída por dados pessoais, exame clínico, fatores de risco, tempo de internação, motivo da alta e destino do paciente ao sair da Clínica de Neurociências.

Os procedimentos foram realizados após parecer favorável do Comitê de Ética da UESB, Protocolo $n^{\circ} 611.914$, atendendo os aspectos éticos constantes na Resolução 466/12 do Conselho Nacional de Saúde. Os dados coletados foram organizados e tabulados em um banco de dados do Programa Estatístico SPSS versão 20.0 por meio da análise descritiva.

\section{RESULTADOS}

A Clínica de Neurociências apresentou no período de dezembro de 2011 a dezembro de 2012, 135 internações de pessoas idosas, com média de idade igual a $77,23( \pm 10,28)$ anos. Destes idosos, os maiores percentuais encontrados foram $57,7 \%$ do sexo feminino, faixa etária entre 70 a 79 anos $(32,6 \%)$ e estado civil casado (a) $(54,1 \%)$, conforme demostrado na tabela 1. 
Tabela 1 - Distribuição dos idosos hospitalizados na Clínica de Neurociências segundo sexo, estado civil e faixa etária dos. Jequié/BA, 2014

\begin{tabular}{|c|c|c|c|c|c|c|}
\hline \multirow[b]{2}{*}{ FAIXA ETÁRIA } & \multicolumn{2}{|c|}{ MASCULINO } & \multicolumn{2}{|c|}{ FEMININO } & \multicolumn{2}{|c|}{ TOTAL } \\
\hline & $\mathrm{N}$ & $\%$ & $\mathbf{N}$ & $\%$ & $\mathbf{N}$ & $\%$ \\
\hline 60 a 69 anos & 16 & 28,1 & 20 & 25,6 & 36 & 26,7 \\
\hline 70 a 79 anos & 20 & 35,1 & 24 & 30,8 & 44 & 32,6 \\
\hline 80 a 89 anos & 16 & 28,1 & 21 & 26,9 & 37 & 27,4 \\
\hline 90 a 99 anos & 5 & 8,8 & 10 & 12,8 & 15 & 11,1 \\
\hline 100 e + anos & - & - & 3 & 3,8 & 3 & 2,2 \\
\hline \multicolumn{7}{|l|}{ Estado Civil } \\
\hline Solteiro (a) & 3 & 5,3 & 6 & 7,8 & 9 & 6,7 \\
\hline Casado (a) & 45 & 78,9 & 28 & 35,9 & 73 & 54,1 \\
\hline Viúvo (a) & 4 & 7,0 & 36 & 46,1 & 40 & 29,6 \\
\hline Não Informado (a) & 5 & 8,8 & 8 & 10,2 & 13 & 9,60 \\
\hline
\end{tabular}

No tocante ao percentual de permanência hospitalar, constatou-se que $11,9 \%$ dos idosos utilizaram o serviço hospitalar de neurociências por 16 dias, $10,4 \%$ pelo período de 14 dias, 8,1\% por 11 dias e $6,7 \%$ pelo período de 9 dias. Quanto à alta hospitalar, verificou-se que $55,6 \%$ dos idosos receberam alta por melhora e 32,6\% por óbito. Destes idosos que tiveram alta hospitalar $26,6 \%$ foram encaminhados para Unidades de Saúde da Família,
$20,7 \%$ para o Atendimento Domiciliar e 10,3\% para Fisioterapia.

Com relação aos problemas de saúde mais frequentes na clínica de neurociências em estudo, agrupadas de acordo com o CID 10, 84,4\% dos idosos apresentavam Acidente Vascular Encefálico não especificado, 7,4\% possuíam Acidente Vascular Encefálico Isquêmico e 3,7\% possuíam Acidente Vascular Encefálico Hemorrágico.

Tabela 2 - Distribuição do Diagnóstico apresentado pelos idosos hospitalizados na Clínica de Neurociências conforme o CID 10. Jequié/BA, 2014

\begin{tabular}{lcccccc}
\hline & \multicolumn{2}{c}{ MASCULINO } & \multicolumn{2}{c}{ Feminino } & \multicolumn{2}{c}{ Total } \\
\hline \multicolumn{1}{c}{ Diagnóstico } & N & $\%$ & N & $\%$ & N & $\%$ \\
\hline AVE não Especificado & 49 & 86,0 & 65 & 83,3 & 114 & 84,4 \\
AVE Isquêmico & 4 & 7,0 & 6 & 7,7 & 10 & 7,4 \\
AVE Hemorrágico & 2 & 3,5 & 3 & 3,8 & 5 & 3,7 \\
Epilepsia & - & - & 2 & 2,6 & 2 & 1,5 \\
Crise Convulsiva & - & - & 1 & 1,3 & 1 & 0,7 \\
Paralisia Central & - & - & 1 & 1,3 & 1 & 0,7 \\
AVE/Epilepsia & 1 & 1,8 & - & - & 1 & 0,7 \\
Traumatismo Intracraniano & 1 & 1,8 & - & - & 1 & 0,7 \\
\hline
\end{tabular}

Grande número de pessoas com 60 anos ou mais é portador de múltiplas doenças coexistentes ou comorbidades, em sua maioria de natureza crônica, não-transmissíveis, associadas ou não à limi- 
tações de desempenho decorrentes dessas ou de suas sequelas (RODRIGUES, 2008). No presente estudo verificou-se uma maior distribuição de $\mathrm{Hi}$ - pertensão Arterial Sistêmica (31,9\%), Diabetes Melitus associada à Hipertensão Arterial Sistêmica (13,3\%) e Hipertensão Arterial Sistêmica associada à Cardiopatia $(4,4 \%)$.

Tabela 3 - Distribuição das patologias associadas nos idosos hospitalizados na Clínica de Neurociências. Jequié/BA, 2014

\begin{tabular}{|c|c|c|c|c|c|c|}
\hline \multirow[b]{2}{*}{ Patologias Associadas } & \multicolumn{2}{|c|}{ MASCULINO } & \multicolumn{2}{|c|}{ FEMININO } & \multicolumn{2}{|c|}{ TOTAL } \\
\hline & $N$ & $\%$ & $N$ & $\%$ & $\mathrm{~N}$ & $\%$ \\
\hline HAS & 15 & 36,6 & 28 & 45,2 & 43 & 41,7 \\
\hline Diabetes/HAS & 5 & 12,2 & 13 & 21,0 & 18 & 17,5 \\
\hline AVE/HAS & 2 & 4,9 & 4 & 6,5 & 6 & 5,8 \\
\hline HAS/ Cardiopatia & 2 & 4,9 & 4 & 6,5 & 6 & 5,8 \\
\hline AVE/Diabetes/HAS & 3 & 7,3 & 2 & 3,2 & 5 & 4,9 \\
\hline AVE & 1 & 2,4 & 3 & 4,8 & 4 & 3,9 \\
\hline Broncoaspiração/HAS & - & - & 3 & 4,8 & 3 & 2,9 \\
\hline AVE/ Diabetes & 2 & 4,9 & - & - & 2 & 1,9 \\
\hline Cardiopatia & 1 & 2,4 & 1 & 1,6 & 2 & 1,9 \\
\hline Tabagismo/ HAS & 2 & 4,9 & - & - & 2 & 1,9 \\
\hline AVE/Diabetes/HAS/Cardiopatia & - & - & 1 & 1,6 & 1 & 1,0 \\
\hline Broncoaspiração/Diabetes/HAS & - & - & 1 & 1,6 & 1 & 1,0 \\
\hline Diabetes & 1 & 2,4 & - & - & 1 & 1,0 \\
\hline Diabetes/HAS/Cardiopatia & 1 & 2,4 & - & - & 1 & 1,0 \\
\hline Etilismo/ HAS & 1 & 2,4 & - & - & 1 & 1,0 \\
\hline Neoplasia/HAS & - & - & 1 & 1,6 & 1 & 1,0 \\
\hline $\begin{array}{l}\text { Tabagismo/ Diabetes/ HAS/ } \\
\text { Cardiopatia }\end{array}$ & 1 & 2,4 & - & - & 1 & 1,0 \\
\hline Tabagismo/ Etilismo & 1 & 2,4 & - & - & 1 & 1,0 \\
\hline Tabagismo/ Etilismo/ HAS & 1 & 2,4 & $\mathrm{O}$ & - & 1 & 1,0 \\
\hline Tabagismo/ Neoplasia & 1 & 2,4 & - & - & 1 & 1,0 \\
\hline Tabagismo/Etilismo/AVE/HAS & 1 & 2,4 & - & - & 1 & 1,0 \\
\hline Tabagismo/Etilismo/DPOC/AVE & - & - & 1 & 1,6 & 1 & 1,0 \\
\hline
\end{tabular}

\section{DISCUSSÃO}

O Brasil passa por um importante período de transição demográfica, que é fortemente marcado pela redução da taxa de natalidade e mortalidade, associados ao aumento da expectativa de vida, que está intimamente vinculado à melhora das condições de vida, da educação e da atenção à saúde presta- da aos idosos. Os fatores econômicos, ambientais, científicos e culturais também contribuem para a mudança na faixa etária da população. De acordo com o Censo demográfico de 2010 do IBGE, existem na Bahia 8.450.009 pessoas com 60 anos ou mais. ${ }^{(8,9)}$

Nesse estudo foi verificado uma prevalência maior de idosos do sexo feminino, fato este que 
pode estar ligado à maior longevidade das mulheres, que tem sido atribuída à menor exposição aos fatores de risco, como tabagismo e etilismo, e à atitude das mesmas em relação às doenças e incapacidades, já que há uma maior procura pelos serviços de saúde por parte das mulheres. O envelhecimento apresenta formas diferenciadas entre os sexos: embora as mulheres vivam mais que os homens, essas passam por um período maior de debilitação biológica antes da morte. Elas apresentam, dessa forma, uma chance maior do que os homens de sofrer as doenças típicas da velhice, tais como: artrite, diabetes, hipertensão e osteoporose. (10-12) Parece haver uma maior relutância do homem, em diversas faixas etárias, a buscar e engajar-se em atividades relativas à prevenção, diagnóstico e tratamento de problemas físicos e emocionais, sendo possível que estes tenham contato com o sistema de saúde em estado mais avançado de doenças. ${ }^{(13)}$

Com o envelhecimento da população idosa brasileira se viu a importância de empreender uma estruturação dos serviços e programas de saúde responsáveis por atender às demandas emergentes do novo perfil epidemiológico do país. Daí a importância das políticas que garantam a assistência especifica ao idoso, que no Brasil, para os efeitos da lei, são os indivíduos com sessenta anos ou mais. É importante considerar que as necessidades de saúde dos idosos requerem uma atenção específica que pode evitar altos custos para o Sistema de Saúde e, sobretudo, proporcionar melhores condições de saúde a essas pessoas. ${ }^{(14)}$ Recentemente, o Ministério da Saúde incluiu a saúde do idoso como item prioritário na agenda de saúde do País, promulgando uma nova política nacional de saúde da pessoa idosa que objetiva, no âmbito do SUS, garantir atenção integral à Saúde da população idosa, enfatizando o envelhecimento saudável e ativo baseada no paradigma da capacidade funcional, abordada de maneira multidimensional. ${ }^{(15)}$ As novas politicas de saúde do idoso tem como objetivo a promoção do envelhecimento saudável, a manutenção da capacidade funcional, a assistência às necessidades de saúde dos idosos, a reabilitação da capacidade funcional comprometida, a capacitação de recursos humanos, o apoio ao desenvolvimento de cuidados informais, e o apoio aos estudos e pesquisas, cabendo aos gestores do SUS viabilizar o alcance do que é proposto. ${ }^{(5)}$

Ainda não há uma rede adequada de ações voltadas para os idosos no país. ${ }^{(16)} \mathrm{O}$ Estatuto do Idoso e as políticas nacionais voltadas para essa população descrevem os direitos e as obrigações da sociedade e do Estado para com os idosos em todos os âmbitos (habitação, saúde, rede de suporte social e familiar e outros), mas na prática essas leis ainda não são efetivamente cumpridas.

Pesquisas vêm mostrando que, qualquer que sejam os indicadores observados na avaliação da qualidade de saúde da população idosa, eles apontam para maior utilização de serviços e custos maiores, quando comparados com os da população mais jovem. ${ }^{(17-18)}$ As internações hospitalares são mais frequentes e o tempo de ocupação do leito é maior quando comparado a outras faixas etárias. Desta forma, o envelhecimento populacional se traduz em maior carga de doenças na população, mais incapacidades e aumento do uso dos serviços de saúde. Mais consultas levam a maior consumo de medicamentos, mais realização de exames complementares e maior hospitalização. As pessoas no início e particularmente no final da vida apresentam mais problemas de saúde. A grande diferença é que as doenças da faixa jovem são agudas e, portanto, de custo menor, enquanto as dos idosos são crônicas e de alto custo. ${ }^{\left({ }^{15}\right)} \mathrm{A}$ hospitalização de pessoas idosas é um motivo de preocupação para os profissionais de saúde devido aos possíveis riscos, complicações, tempo de internação e custos que podem surgir dessa modalidade de assistência. ${ }^{(17)}$

O curso das doenças crônicas que acometem os idosos é um dos fatores que favorecem um tempo de internação mais longo para esse grupo. Ou seja, as pessoas acima de 60 anos levam um tempo maior para produzir as respostas do seu sistema imunológico, bem como maior tempo para as respostas terapêuticas, em relação aos mais jovens. Isto se deve ao processo normal de envelhecimento, 
que tende a ficar mais comprometido quando essas doenças estão presentes. Pode-se pensar nos riscos e complicações advindas de um período longo de internação, provocados pela imobilidade no leito, como é caso da formação de úlceras de decúbito, piora das condições respiratórias, exposição maior às infecções hospitalares e ansiedade pelo afastamento familiar, que podem agravar ainda mais o quadro clínico dos idosos. Período prolongado de internação concorre para o estabelecimento de incapacidades, maior exposição a risco de infecção hospitalar, entre outras complicações que podem refletir na recuperação das pessoas idosas. ${ }^{(17)}$

A alta deve ser precoce, porém não precipitada, observando-se o momento em que as possibilidades da unidade foram exauridas, e então o caso deve ser encaminhado ao domicílio ou outro nível assistencial adequado, evitando tanto os riscos da hospitalização, como a progressiva dificuldade de adaptação ao seu meio. ${ }^{(18)}$

Os agravos decorrentes das doenças crônicas não transmissíveis têm sido as principais causas de óbito na população idosa, seguindo uma tendência mundial. Quando são analisadas as causas específicas, a doença cerebrovascular ocupa o primeiro lugar em mortalidade no país, tanto em idosos quanto na população geral, e as doenças cardiovasculares, o segundo lugar. Nos países de alta renda e no mundo de uma forma geral, observa-se o inverso quanto a essas duas causas, ou seja, doenças cardiovasculares, em primeiro, e doença cerebrovascular, em segundo. Vários motivos estão implicados nessa discrepância em relação ao restante do mundo, provavelmente um dos mais importantes seja a alta prevalência de hipertensão arterial na população brasileira e o não tratamento ou o tratamento inadequado dessa doença, tendo em vista que a hipertensão arterial é o principal fator modificável da doença cerebrovascular. ${ }^{(19)}$

A hipertensão arterial é uma realidade crescente na população, principalmente nos idosos, e que pode levar a sérios danos à saúde. HAS leva ao acometimento isquêmico cardíaco, renal, vascular periférico e cerebral. $\mathrm{O}$ acidente vascular encefáli- co, uma das complicações da hipertensão arterial, é uma das principais causas de óbitos no Brasil, e também pode levar a incapacidades funcionais, devido a complicações neurológicas e motoras. ${ }^{(20)}$ No contexto da geriatria, as doenças neurológicas assumem maior relevância devido à importante relação entre idade e incidência. Embora essas patologias possam ocorrer em todas as faixas etárias, sabe-se que há aumento significativo de sua incidência a partir da sexta década de vida, praticamente dobrando a ocorrência a cada nova década. Isto pode estar relacionado, dentre outros fatores, à presença de distúrbios das funções sensoriais, bem como à senescência dos sistemas neuromusculares e da função esquelética entre a população idosa. ${ }^{(19)}$

O fenômeno do envelhecimento populacional favoreceu o aparecimento de polipatologias crônicas, a exemplo do Acidente Vascular Encefálico (AVE). O AVE é uma das causas mais comuns de morte e invalidez permanente, tendo uma participação efetiva no número de internamentos, comprometendo em muito a qualidade de vida de todos. ${ }^{(18)}$

O termo acidente vascular encefálico é usado para designar o déficit neurológico (transitório ou definitivo) em uma área cerebral secundário a lesão vascular, e representa um grupo de doenças com manifestações clínicas semelhantes, mas que possuem etiologias diversas: AVE hemorrágico compreende a hemorragia subaracnóide, em geral decorrente da ruptura de aneurismas saculares congênitos localizados nas artérias do polígono de Willis e a hemorragia intraparenquimatosa, cujo mecanismo causal básico é a degeneração hialina de artérias intraparenquimatosas cerebrais, tendo como principal doença associada a hipertensão arterial sistêmica; AVE isquêmico descreve o déficit neurológico resultante da insuficiência de suprimento sanguíneo cerebral, podendo ser temporário (episódio isquêmico transitório) ou permanente, e tendo como principais fatores de risco a HAS, as cardiopatias e o diabetes mellitus. Outras etiologias podem estar associadas ao AVE, tais como coagulopatias, tumores, arterites inflamatórias e infecciosas. ${ }^{(19)}$ 
O AVE afeta cerca de 600.000 pessoas a cada ano, com estimativa de quatro milhões de sobreviventes apresentar alguma disfunção motora. Sua incidência aumenta com a idade, dobrando a cada década após os cinquenta e cinco anos. O AVE está relacionado ao avanço da idade, associado com fatores como: hipertensão arterial, diabetes, uso de anticoncepcionais, alcoolismo, tabagismo, sedentarismo. Como prevenção primária, a principal intervenção seria diminuir ou abolir os fatores de risco para a ocorrência desta patologia. Esses fatores podem ser divididos em não modificáveis (idade, sexo, raça e história familiar) e modificáveis (hipertensão arterial, diabetes mellitus, tabagismo, obesidade, vida sedentária, uso de álcool, uso de anticoncepcionais, uso de drogas, enxaqueca e dislipidemias), sendo estes últimos considerados os mais importantes, pois são passíveis de intervenção. ${ }^{(20)}$ Dentre os fatores modificáveis, o principal fator de risco é a hipertensão arterial (HA), pois indivíduos com pressão arterial sistólica maior que $160 \mathrm{~mm} \mathrm{Hg}$ e diastólica maior que $95 \mathrm{mmHg}$ possuem risco aumentado para o AVE de quatro vezes em relação à população em geral. ${ }^{(19)}$ Estas informações podem servir como explicação para a alta prevalência do AVE entre as patologias neurológicas neste estudo, representando $95,5 \%$ dos casos.

\section{CONCLUSÃO}

Constatou-se que houve uma maior frequência de idosos do sexo feminino, faixa etária entre 70 a 79 anos e estado civil casado(a). No tocante ao percentual de permanência hospitalar, constatou-se que o maior tempo de permanência do ser no serviço hospitalar de neurociências foi de 16 dias. Quanto à alta hospitalar, verificou-se a maioria dos idosos recebeu alta por melhora, destes idosos que tiveram alta hospitalar a maior parte foi encaminhada para Unidades de Saúde da Família.

Com relação aos problemas de saúde mais frequentes na clínica de neurociências em estudo, agrupadas de acordo com o CID 10, verificou-se uma maior distribuição de Hipertensão Arterial
Sistêmica, Diabetes Melitus associada à Hipertensão Arterial Sistêmica e Hipertensão Arterial Sistêmica associada à Cardiopatia.

Nesta perspectiva, reafirma-se aqui a necessidade de estudos sobre a população idosa do Brasil e do mundo, como também a busca por capacitação profissional especifica associada à melhor execução das politicas de saúde.

\section{REFERÊNCIAS}

1. Rodrigues CC, Ribeiro RCH. Perfil epidemiológico dos idosos atendidos na emergência de um hospital escola. Arq Ciênc Saúde. 2012;19(2):37-41.

2. Tannure MC, Alves M, Sena RR, Chianca TCM. Perfil epidemiológico da população idosa de Belo Horizonte-MG, Brasil. Rev Bras Enferm. 2010;63(5):817-22.

3. Moraes EN, Moraes FL, Lima SPP. Características biológicas e psicológicas do envelhecimento. Rev Méd Minas Gerais. 2010;2O(1):67-73.

4. Parreira JG, Solda SC, Perlingeiro JAG, Padovese CC, Karakhanian WZ, Assef JC. Análise comparativa das características do trauma entre pacientes idosos e não idosos. Rev Assoc Méd Bras. 2010;56(5):541-6.

5. Álvares LM, Lima RC; Silva RA. Ocorrência de quedas em idosos residentes em instituições de longa permanência em Pelotas, Rio Grande do Sul, Brasil. Cad. Saúde Pública. 2010;26(1):3140.

6. Silva $M C L$ et al. Caracterização clínica e motora-funcional de idosos hospitalizados pósAcidente Vascular Cerebral. Rev Neurocienc. 2014;22(3):337-343.

7. Jaul E. Assessment and management of pressure ulcers in the elderly: current strategies. Drugs Aging. 2010;27(4):311-25.

8. Oliveira N, Reis LA. Caracterização das ulceras de pressão em idosos hospitalizados. Revista Enfermagem Contemporânea. 2013;2(1):146-156.

9. Instituto Brasileiro de Geografia e Estatística. Censo Demográfico 2010: Características da 
população - amostra. [acesso em 26 de mar de 2014]. Disponível em: http://www.ibge.gov.br/ estadosat/temas.php?sigla=ba\&tema=censode mog2O1O_amostra

10. Schein LE, César JA. Perfil de idosos admitidos em unidades de terapia intensiva gerais em Rio Grande, RS: resultados de um estudo de demanda. Rev Bras Epidemiol. 2010; 13(2):289301.

11. Rabelo LPO et al. Perfil de idosos internados em um hospital universitário. Rev Min Enferm. 2010;14(3):293-300.

12. Veras R. Envelhecimento populacional contemporâneo: demandas, desafios e inovações. Rev Saúde Pública. 2009;43(3):548-54.

13. Depolito C, Leocadio PLLF, Cordeiro RC. Declínio funcional de idosa institucionalizada: aplicabilidade do modelo da Classificação Internacional de Funcionalidade, Incapacidade e Saúde. Fisioter Pesqui. 2009;16(2):183-189.

14. Lira LN, Santos SSC, Gautério DP et al. Histórico de enfermagem para idosos hospitalizados: base para diagnósticos e prescrições. Rev Enferm UFPE on line. 2013; 7(8):5198-206.
15. Motta CCR, Hansel CG, Silva J. Perfil de internações de pessoas idosas em um hospital público. Rev Eletrônica Enferm. 2010;12(3):471-7.

16. Castro VC et al. Perfil de internações hospitalares de idosos no âmbito do sistema único de saúde. Rev Rene. 2013;14(4):791-800.

17. Duca GFD, Nader GA, Santos IS, Hallal PC. Hospitalização e fatores associados entre residentes de instituições de longa permanência para idosos. Cad Saúde Publica. 2010; 26(7):1403-10.

18. Sthal HC, Berti HW, Palhares VC. Caracterização de idosos internados em enfermaria de pronto-socorro quanto à vulnerabilidade social e programática. Esc Anna Nery Ver Enferm. 2O10; 14(4):697-704.

19. Furuya RK, Birolim MM, Biazin DT, Rossi LA. A integralidade e suas interfaces no cuidado ao idoso em unidade de terapia intensiva. Rev Enferm UERJ. 2O11;19(1):158-62.

2O. Caldas CP, Teixeira PC. O idoso hospitalizado sob o olhar da teoria de enfermagem humanística. Cienc Cuid Saude. 2012;11(4):748-757. 